\title{
Mesure et contrôle de très faibles débits Le Picoflow
}

\author{
Yves Lecoffre \\ Hélispire ( $\left.{ }^{1}\right)$
}

\section{Les besoins}

L'utilisation de très faibles débits de gaz ou de liquides, typiquement inférieurs à $1 \mathrm{~cm}^{3}$ par minute, se rencontre dans de nombreuses applications industrielles, domestiques, agricoles ou médicales dont il est difficile d'établir une liste en raison de leur très grande diversité.

Pour les débits couramment utilisés dans l'industrie, milieu dans lequel on parle rarement de débits inférieurs à $1 \mathrm{l} / \mathrm{s}$, les solutions de mesure et de contrôle des débits sont légion: méthodes optiques, électromagnétiques, mécaniques, à ultrasons, à effet Coriolis, ou, plus prosaïquement les appareils déprimogènes permettent de répondre, avec une bonne précision, aux besoins en débitmétrie ; les systèmes de contrôle de débit mettant en jeu des vannes ou des pompes à vitesse variable sont parfaitement bien maîtrisés.

La mise en œuvre de ces procédés classiques devient très délicate lorsque les débits diminuent. Une vanne traditionnelle permettant de réguler un débit d'eau de $1 \mathrm{~cm}^{3}$ par jour sous une différence de pression de 1 bar aurait un diamètre de $1 \mu \mathrm{m}$. Outre la quasi-impossibilité de réaliser mécaniquement une vanne de réglage de cette dimension, il est facile d'imaginer les problèmes liés à la propreté des fluides et des circuits.

Les seules solutions pratiques pour réaliser ces performances consistaient à utiliser des pompes volumétriques, et, en particulier, des seringues motorisées ou des micropompes doseuses qui mettent en œuvre des mécaniques assez délicates et sophistiquées dont l'application est limitée à des usages spéciaux.

Il était intéressant de rechercher des solutions variantes à ces procédés et de tenter de créer une hydraulique des micro-débits en conduite permettant de réaliser la plupart des fonctions actuellement couvertes par l'hydraulique

(1) Hélispire, Allée des Dauphins, 38330 Saint Ismier. Tél. : (33) 76523934. traditionnelle dans le domaine de la débitmétrie, du contrôle des débits, des appareils de sécurité, des mélanges ou des transferts d'énergie par pompage ou turbinage.

La société Hélispire, à partir des appareils Picoflow, couvre cette gamme d'applications nouvelles, tout particulièrement dans le domaine de la débitmétrie, du contrôle et du dosage. On contrôle les fluides newtoniens, mais également les fluides comportant des particules en suspension ou présentant des rhéologies complexes.

\section{Les principes de base}

La vanne que nous avons évoquée ci-dessus, de diamètre égal à $1 \mu$, permet de contrôler un débit de $1 \mathrm{~cm}^{3} / \mathrm{J}$ sous une différence de pression de 1 bar.

Pour réaliser la même fonction avec de l'eau dont la viscosité dynamique est de $10^{-3} \mathrm{~Pa}$.s, on peut utiliser un tube capillaire de $10 \mathrm{~m}$ de longueur et de $82,5 \mu \mathrm{m}$ de diamètre. On conçoit que les risques de bouchage associés à ce composant sont beaucoup moins importants que dans le cas précédent.

Pour contrôler le même débit d'air, le diamètre du capillaire de $10 \mathrm{~m}$ de longueur serait de $30 \mu \mathrm{m}$, valeur qui reste raisonnable et pratiquement réalisable.

La perte de charge d'un tel tube capillaire de section circulaire, siège d'un écoulement laminaire de fluide Newtonien incompressible, est donnée par :

$$
\Delta p=\frac{128 \mu L Q}{\pi D^{4}} .
$$

$\Delta p$ est proportionnelle à la longueur du tube. Il suffit donc de faire varier la longueur de celui-ci pour modifier son débit et donc son coefficient de perte de charge.

Pour un fluide compressible, on calcule cette perte de charge à partir de l'équation différentielle suivante :

$$
\Delta p=\int_{0}^{L} \frac{128 \mu Q}{\pi D^{4}} \mathrm{~d} x .
$$

Bien entendu, en modifiant la différence de pression aux bornes du composant, on fait varier proportionnellement le

\section{Measurement and control of very small flows. Picoflow}

The very small flows are difficult to measure. With Picoflow, Hélispire aims at covering new applications, including fluids with complex rheology as well as newtonian fluids. 
débit dans le cas d'un fluide incompressible. Cette dépendance linéaire de la perte de charge et du débit est intéressante, car la gamme d'utilisation relative d'une telle perte de charge laminaire est, à précision égale, supérieure à celle d'une perte de charge quadratique traditionnelle.

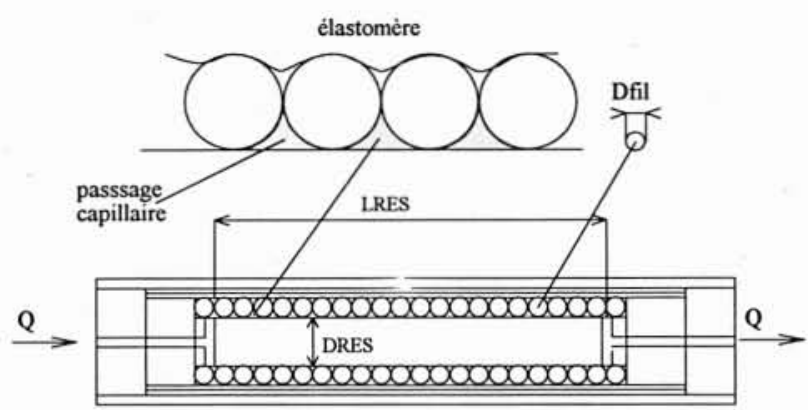

1. Picospring, mode de réalisation possible du Picoflow.

Les propriétés de ce type d'écoulement sont remarquables. La difficulté essentielle pour l'utiliser est liée à la réalisation de tubes capillaires très précis de très grande longueur.

Plusieurs solutions ont été développées par Hélispire, parmi lesquelles le système «Picospring », illustré sur la figure 1. On utilise un ressort à boudin à spires jointives, montré serré sur un axe cylindrique. La zone vide existant entre le cylindre et le ressort constitue un canal hélicoïdal de très grande longueur. Sa dimension est parfaitement contrôlée, en raison des très bonnes tolérances de fabrication des fils à ressorts.

Le diamètre hydraulique équivalent de ce passage est environ trois fois inférieur au diamètre du fil. Il est donc facile de créer un canal de $80 \mu \mathrm{m}$ en utilisant un ressort dont le diamètre de fil est de $240 \mu \mathrm{m}$. Quant à la longueur de $10 \mathrm{~m}$, on la réalise aisément en utilisant un cylindre de $20 \mathrm{~mm}$ sur une longueur de $38 \mathrm{~mm}$ correspondant à 160 tours de fil.

La figure ci-dessous représente un Picoflow industriel, muni à l'entrée d'un filtre intégré en métal fritté.

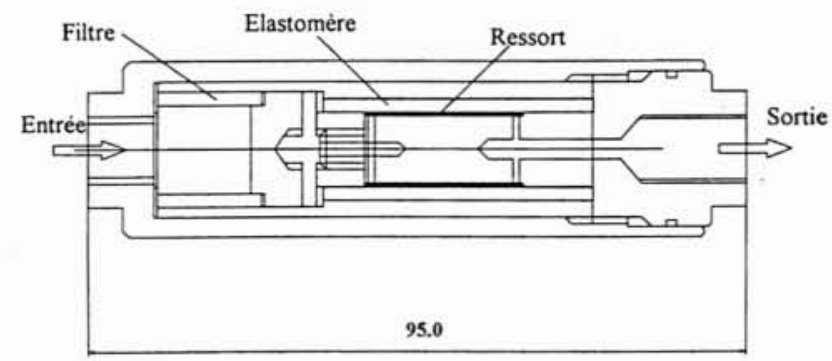

2. Picoflow à filtre intégré.

D'autres astuces constructives de ce type ont été mises en œuvre pour réaliser des Picoflow, le choix de l'une ou l'autre d'entre elles étant fonction des applications envisagées et des matériaux utilisés. On utilise toujours des canaux formés par contact entre deux pièces mécaniques.
Dans tous les cas, le capillaire est nettoyable et stérilisable, quelle que soit sa longueur ; c'est l'un des critères essentiels pour un tel dispositif.

\section{Gamme de fonctionnement}

Le graphique 3 donne la gamme de fonctionnement des appareils Picoflow. Les débits sont fonction de la différence de pression aux bornes du capillaire, de son diamètre équivalent et de sa longueur. Le fluide de référence est de l'eau dont la viscosité cinématique de référence est égale à :

$$
\mu=10^{-3} \text { Pa.s . }
$$

Pour passer aux performances avec un autre fluide, il suffit de diviser le débit d'eau par le rapport de la viscosité dynamique de l'eau à celle du fluide. On obtient alors le débit du fluide considéré sous la même différence de pression.

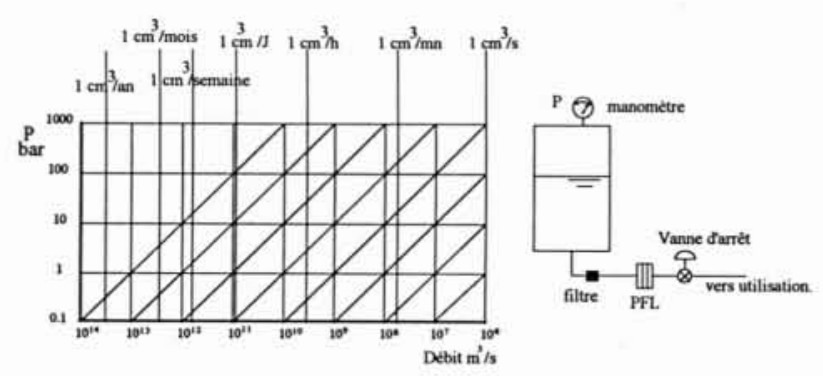

3. Gamme d'utilisation des Picoflow.

Les différentes droites inclinées correspondent chacune à un Picoflow particulier, allant d'un diamètre de $1 \mathrm{~mm}$ pour le plus gros à un diamètre de $30 \mu \mathrm{m}$ pour celui qui permet d'obtenir des débits inférieurs à $1 \mathrm{~cm}^{3} / \mathrm{an}$.

\section{Débitmétrie}

\subsection{Les principes}

La mesure de débits par Picoflow résulte simplement de l'application de la formule 1.

Dans le cas d'un liquide, on mesure la température afin de connaître la viscosité à partir de tables. Cette fonction et le calcul de débit correspondant sont, en général, automatisés.

L'étalonnage de l'appareil se fait en utilisant un fluide quelconque, à la seule condition qu'il soit Newtonien et que le régime d'écoulement soit laminaire. Il peut être, dans certains cas, intéressant d'effectuer les étalonnages au moyen d'un fluide stérile ou désinfectant.

Dans le cas de gaz, on doit considérer deux situations :

- lorsque le rapport de la perte de charge à la pression aval est faible, cas par exemple de la débitmétrie de gaz sous haute pression, on peut assimiler le gaz à un fluide incompressible et revenir au cas précédent ; 

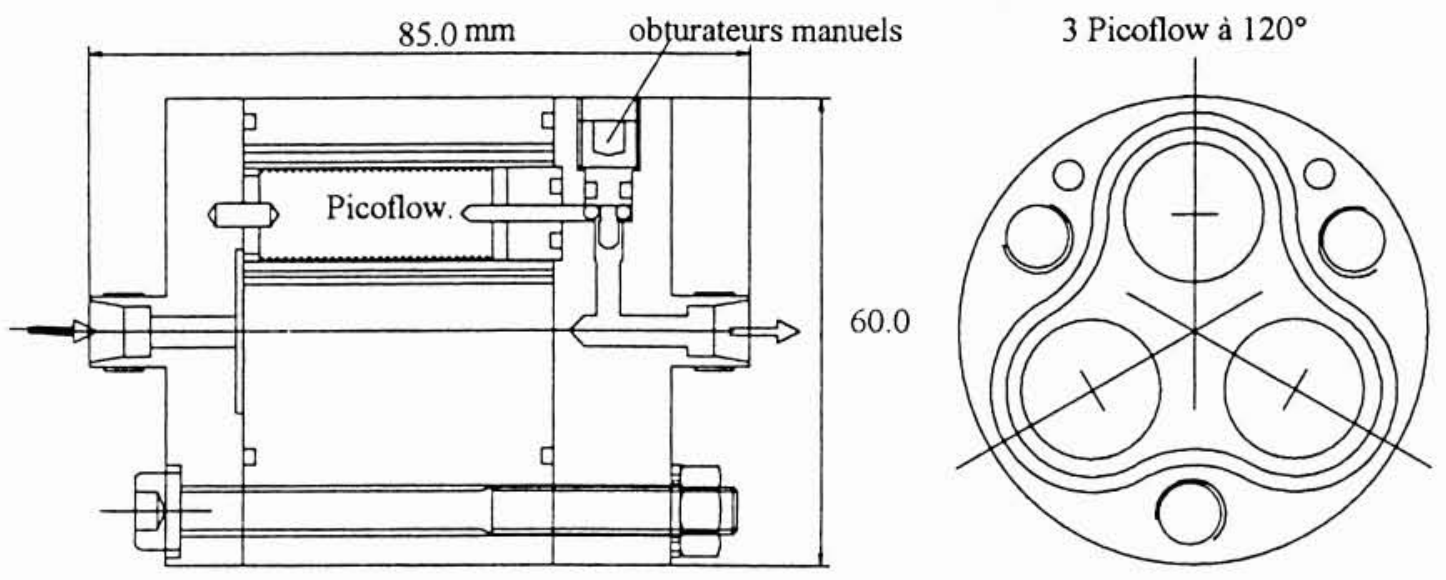

\section{Débitmétrie à ammoniac. Applications spatiales.}

- lorsque la perte de charge n'est plus négligeable, on doit intégrer l'équation ci-dessus en tenant compte du comportement thermodynamique du gaz, qui peut être adiabatique, isotherme ou polytropique. Pour des mesures de grande précision, on utilise des échangeurs de chaleur qui imposent au gaz une détente isotherme.

On peut, selon cette dernière hypothèse, et pour un gaz parfait, calculer le débit masse de fluide passant dans la canalisation :

$$
w=\Delta p \frac{p_{\mathrm{am}}+p_{\mathrm{av}}}{2} \frac{\pi D^{4}}{128 \mu L} \frac{1}{\frac{R}{M} T}
$$

$p_{\text {am }}$ et $p_{\text {av }}$ correspondant aux conditions amont et aval, $T$ étant la température absolue, $R$ la constante des gaz parfaits et $M$ la masse molaire du gaz considéré.

\subsection{Applications}

Les applications des Picoflow en débitmétrie concernent les très faibles débits pour lesquels il n'existe pas de solutions satisfaisantes et bon marché.

Citons un appareil réalisé pour le CENG destiné à mesurer des débits d'ammoniac liquide dans l'espace dans une gamme comprise entre 0,05 et $0,24 \mathrm{~g} / \mathrm{s}$.

Hélispire a réalisé un débitmètre compact intégrant trois Picospring et muni de deux vannes d'arrêt. L'ensemble, représenté sur la figure 4 permet de couvrir la gamme spécifiée.

Dans une autre application relative au dosage de microdébits d'huile animale installée sur une ligne de production de verre, on a réalisé un système redondant comportant un doseur à base de Picoflow, doublé d'une mesure en ligne basée sur le même principe.

Hélispire développe également des débitmètres universels permettant de couvrir des gammes particulièrement étendues par mise en service de plusieurs Picoflow et d'un seul capteur différentiel. On peut, par exemple, mesurer des débits d'eau compris entre $1 \mathrm{~cm}^{3}$ par an et $1 \mathrm{~cm}^{3}$ par minute avec seulement quatre Picoflow, soit une dynamique de 500000 . L'appareil est facile à automatiser au moyen de vannes tout ou rien.

\section{Le dosage}

La seconde famille d'application des Picoflow concerne le développement de systèmes de dosage performants et bon marché, pouvant répondre à des besoins très particuliers, automatisables ou non.

Hélispire développe actuellement deux gammes de produits, l'une destinée au grand public, dont le premier représentant est le Picodrop, l'autre, plus industrielle, baptisée Picodose.

\subsection{Le Picodrop}

Les appareils de diffusion de fluides odorants ou antiseptiques dans l'habitation sont presque tous basés sur l'utilisation de mèches ou de milieux poreux imbibés du produit. De par leur principe même, ces systèmes privilégient la diffusion des espèces les plus volatiles. Il en résulte que l'odeur est trop intense pendant les premiers jours de fonctionnement puis s'atténue jusqu'à pratiquement disparaître alors qu'il reste beaucoup de solvant. De plus, on constate que le débit est fortement fonction de la température du milieu récepteur.

Pour éviter ces inconvénients, Hélispire a développé un appareil original à base de Picoflow, le Picodrop, qui fonctionne par vidange gravitaire au travers d'une perte de charge laminaire.

La figure 5 donne un exemple d'un tel appareil. La disposition particulière de la prise d'air permet d'imposer

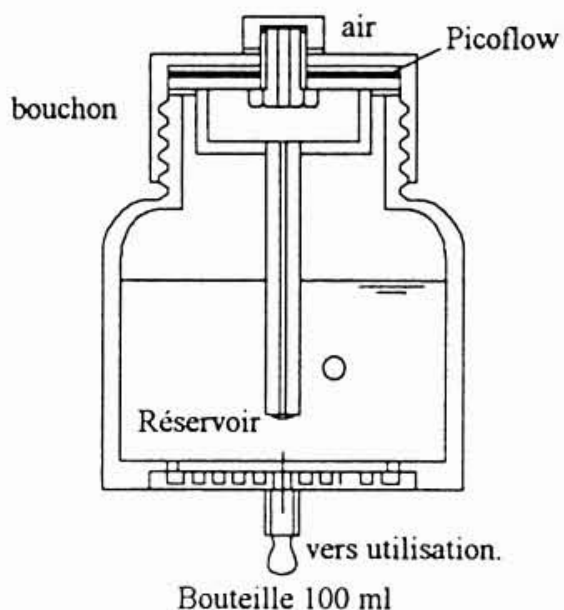

5. Picodrop, diffuseur de parfums. 
une pression constante dans le réservoir indépendamment du niveau de liquide. Le débit de liquide peut donc être maintenu constant.

Hélispire développe une gamme de produits basés sur ce principe. Le débit nominal des produits de très grande série est de $100 \mathrm{ml}$ par mois. Les produits sont conditionnés dans des « éco-recharges » que l'on clique simplement sur l'étage technique. Le fluide est, en général, répandu sur un support en cellulose qui permet sa diffusion en raison de sa surface d'échange.

\subsection{Applications agricoles}

L'appareil peut être utilisé dans des applications nécessitant un contrôle rustique et précis, éventuellement réglable, de très faibles débits pendant une longue période.
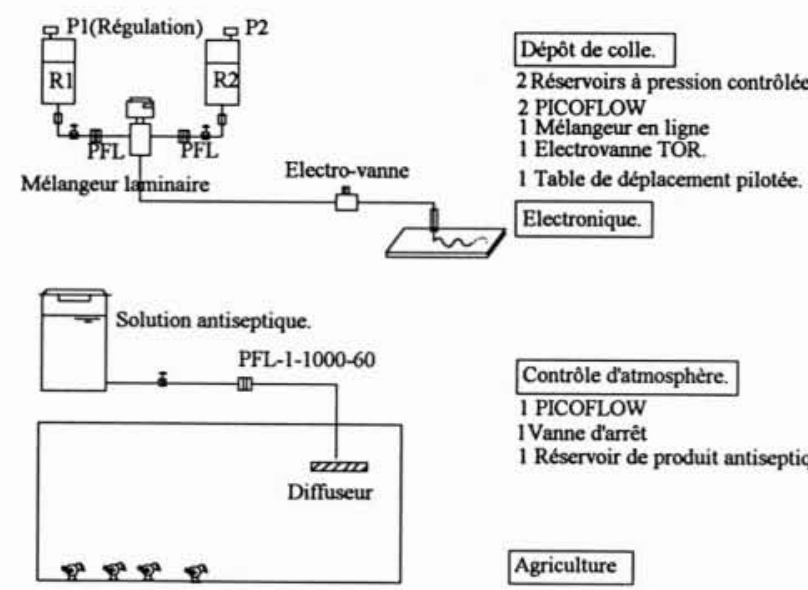

1 PICOFLOW

1 Vanne d'arrêt

1 Réservoir de produit antiseptique.

2)

\section{Agriculture}

6. Deux applications du Picoflow.

Un exemple, schématisé sur la figure 6, en est la stérilisation au formol des œufs dans les couveuses pour éviter la salmonellose. On dispose simplement un réservoir contenant une solution de formol au-dessus de la couveuse. On place un Picoflow sur une tuyauterie qui pénètre dans la couveuse dont la température est constante. Il suffit de faire varier, si besoin, la hauteur entre les points A et B correspondant respectivement à la surface libre du réservoir et au point d'injection pour adapter le débit, qui lui est proportionnel.

Les applications de ce type sont multiples dans le domaine des engrais, des insecticides ou des produits antiseptiques.

\subsection{Picodose, le doseur universel}

Les systèmes de mécaniques microdosage par utilisation de pompes à déplacement positif, seringues motorisées ou pompes volumétriques, peuvent poser un certain nombre de problèmes, par exemple les suivants :

- la gestion des glissements mécaniques et les problèmes d'étanchéité associés ;

- l'apparition de petites instabilités résultant de la non constance des coefficients de frottement entre le piston et le cylindre ;
- la compatibilité de matériaux devant assurer des fonctions mécaniques avec des critères de pureté ou d'agressivité chimique des produits à transférer ;

- la difficulté à travailler dans des conditions agressives de pression, de température, d'humidité ou d'environnement général.

Pour éviter ces problèmes, Hélispire a développé un système de dosage universel, baptisé « Picodose ", dont les performances sont les suivantes :

- gamme de fonctionnement pratiquement illimitée, pouvant atteindre $10^{6}$;

- pas de pièces mécaniques mobiles en contact avec le fluide à doser ;

- débit régulé, constant ou variable, sans aucun à-coup ;

- fonctionnement avec tous les fluides, liquides ou gazeux et certains fluides diphasiques, par exemple des liquides chargés ;

— conteneurs en matériaux indifférents.

L'appareil dont le schéma est donné sur la figure 7 est muni d'un micro-contrôleur qui pilote la pression dans le fluide à doser à partir de la mesure de débit réalisée par l'un des Picoflow de la batterie de mesure. Le choix de l'un ou l'autre de ces Picoflow est fonction du débit à réguler.

Pour doser des fluides dangereux, ultrapurs, chargés ou à rhéologie complexe, on utilise un fluide intermédiaire. Le fluide à doser est inclus dans un réservoir déformable et poussé par un fluide intermédiaire, par exemple un liquide dont on régule la pression. Il suffit alors de mesurer le débit du fluide propulseur pour connaître celui du fluide propulsé. Hélispire développe actuellement cette dernière application pour le dosage de graisses végétales dans l'industrie cosmétique, ces graisses ayant une rhéologie particulièrement complexe et éminemment variable.

\section{Organes de sécurité : Le «Clapico »}

Les premières études sur l'utilisation des Picoflow concernaient la distribution de fluides en des points multiples de réseaux de grandes longueurs. Un exemple peut en être l'arrosage des plantes en pots dans des serres ou l'alimentation en produits divers de cultures biologiques dans des tubes à essais. Ce type de réseau, en général ramifié, pose un problème majeur de sécurité : la rupture d'une canalisation. Le débit pourrait alors atteindre des valeurs inacceptables pouvant mettre en péril les locaux ou engendrer des inconvénients sur le personnel.

Pour éviter ces problèmes, Hélispire a développé un clapet qui se ferme définitivement lorsque le débit dépasse une certaine valeur, légèrement supérieure à la somme des débits individuels des appareils situés à son aval. L'appareil se nomme «Clapico». Il contient, bien entendu, comme élément de base, un Picoflow calibré. On peut le placer en tête de branches secondaires dans le cas d'une distribution en réseau ramifié.

\section{Applications à la débitmétrie industrielle}

Picoflow est un débitmètre laminaire, adapté à la mesure de faibles débits. On peut cependant l'utiliser, associé à un 


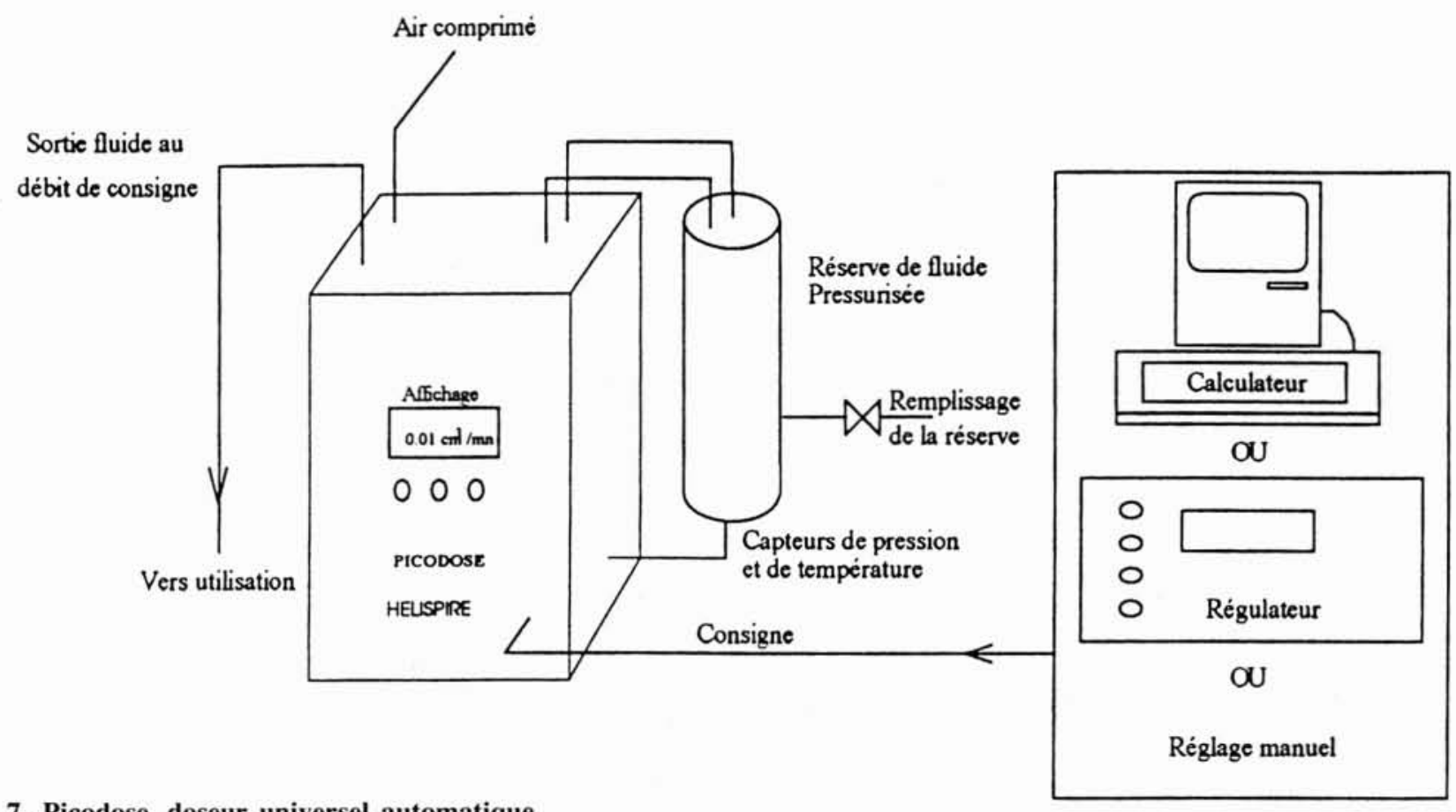

7. Picodose, doseur universel automatique.

mélangeur efficace, pour mesurer des débits très importants par la méthode des marqueurs.

Le principe consiste à injecter à l'amont du mélangeur un débit parfaitement connu d'un marqueur convenable, nucléaire ou chimique, par exemple, à mélanger le marqueur dans l'écoulement principal, puis à doser le marqueur dans le flux sortant du mélangeur. La difficulté rencontrée dans ce type de mesure est que les mélangeurs en ligne classiques sont peu efficaces et ne permettent pas d'obtenir des mélanges spatialement homogènes dans la conduite aval.

Le mélangeur Optimix, développé par Ylec Consultants et récemment testé au CENG dans le cadre d'une action aidée par le MRT, permet de réaliser ce processus de manière très efficace. On peut en envisager l'utilisation dans des tuyauteries de grand diamètre ou pour effectuer la débitmétrie de fluides difficiles à mesurer avec des techniques traditionnelles fonctionnant en eau froide.

\section{Conclusion}

Les systèmes brevetés Picoflow apportent une réponse nouvelle aux problèmes de mesure et de dosage de très faibles quantités de fluides. Leurs applications potentielles en mesures et dosages couvrent des domaines nombreux, chimie, agriculture, cosmétique, parfumerie, médecine, pharmacie. Ils se déclinent sur une variété de marchés allant du grand public aux domaines de la haute technologie, spatial ou médecine par exemple.

Parmi les produits actuellement commercialisés par Hélispire figurent le Picodrop, utilisé dans la diffusion de parfums, les débitmètres divers et les doseurs de produits fluides dont le nom générique est Picodose. Certains de ces appareils utilisent un fluide intermédiaire et permettent le contrôle des débits de fluides agressifs ou à rhéologie complexe.

Cet appareil peut être associé à d'autres composants pour intéresser les domaines des écoulements industriels classiques. Citons le mariage de Picoflow et des mélangeurs Optimix, ou encore les procédés d'injection de colorants dans les installations d'essais hydrodynamiques.

D'autres produits de grande diffusion sont en cours de développement chez Hélispire, aussi bien dans le domaine grand public que dans celui des technologies avancées. L'un des objectifs est de promouvoir l'utilisation des microdébits dans des environnements et pour des utilisations inhabituels. 